\title{
Numerical Study of Cooling by Ferrofluids in an Electrical Transformer using an Axisymmetric Model
}

\author{
Raphaël Zanella ${ }^{1,2}$, Xavier Mininger ${ }^{2}$, Caroline Nore ${ }^{1}$, Frédéric Bouillault ${ }^{2}$, and Jean-Luc Guermond ${ }^{3}$ \\ ${ }^{1}$ LIMSI, CNRS, Univ. Paris-Sud, Université Paris-Saclay, F-91405 Orsay, France \\ ${ }^{2}$ GeePs, CNRS, CentraleSupélec, Univ. Paris-Sud, Université Paris-Saclay, Sorbonne Université, \\ 3 \& 11 rue Joliot-Curie, Plateau de Moulon 91192 Gif-sur-Yvette CEDEX, France \\ ${ }^{3}$ Department of Mathematics, Texas A\&M University, College Station, TX 77843 USA \\ Xavier.Mininger@geeps.centralesupelec.fr, Caroline.Nore@limsi.fr
}

\begin{abstract}
We present a numerical study of the cooling of a $40 \mathrm{kVA}$ electrical transformer by using an oil-based ferrofluid. The temperature and velocity fields are compared to that obtained with regular transformer oil to assess the benefits of the ferrofluid. The interest of ferrofluids is in the thermomagnetic convection phenomenon generated by the leakage magnetic field. An axisymmetric model of the transformer shows that the ferrofluid reduces the maximum temperature.
\end{abstract}

Index Terms-Electrical transformer, ferrofluid, thermomagnetic convection, finite element method.

\section{Problem And Modeling}

Several research groups have proposed to enhance heat transfer in power transformers by taking advantage of the leakage magnetic flux in the insulating liquid [1]-[2]. Their approach relies on the use of oil-based ferrofluid as cooling agent. For this type of fluid a phenomenon called thermomagnetic convection can be strong enough to enhance the mixing effects of thermogravitational convection.

To assess this claim, we perform computations on an electromagnetic system resembling a $40 \mathrm{kVA}(20 \mathrm{kV} / 400 \mathrm{~V})$ transformer. We use an axisymmetric model, see Fig. 1.We do not model the full magnetic circuit since we are only interested in the leakage magnetic field, the Joule effect generated by the coils and the induced natural convection of the liquid. The volume fraction of magnetic nanoparticles in the ferrofluid is of only $1 \%$.

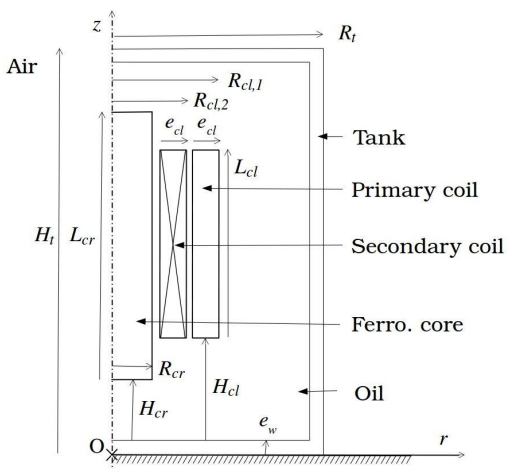

Fig. 1: Transformer model $\left(H_{t}=46 \mathrm{~cm}\right.$, $\left.R_{t}=21.1 \mathrm{~cm}\right)$.

The governing equations consist of the Navier-Stokes equations augmented with the Boussinesq approximation and a magnetic body force (Kelvin force model here), the temperature equation and Maxwell's equations. A similar set of equations is described in [3]. On the tank walls, we use Robin boundary conditions for the temperature with a high convection coefficient to model the presence of heat transfer fins. The equations are solved with the SFEMaNS finite element code [3].

\section{RESULTS}

Fig. 2 compares the temperature fields and velocity field streamlines at quasi-steady regime in a meridian plane (revolution axis on the left). Results with regular transformer oil are shown on the left and results with the ferrofluid are shown on the right. Using ferrofluid reduces the maximum temperature in the primary coil by $2.2^{\circ} \mathrm{C}$ and in the secondary coil by $5^{\circ} \mathrm{C}$. Near the coil bottom, the streamlines show that the magnetic force reinforces the convection pattern entailing a temperature homogenization and a decrease of the maximum temperature. The main limit of this setup is that the coils are superimposed: the magnetic fields generated by the coils, carrying opposite currents, almost cancel each other everywhere except close to the coils. Other transformer geometries should be tested to improve the thermomagnetic convection effect. More details on the modeling and results are available in [4, pp. 137-154].

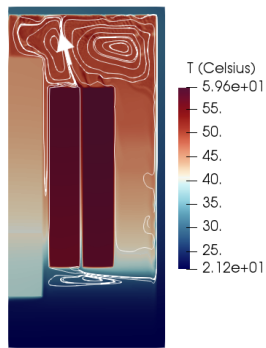

(a) Regular transformer oil

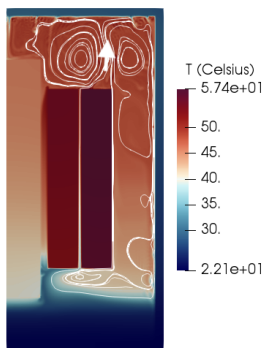

(b) Ferrofluid
Fig. 2: Temperature field $\left({ }^{\circ} \mathrm{C}\right)$ at $t=20000 \mathrm{~s}$ and velocity field streamlines. The arrow represents the flow direction.

\section{REFERENCES}

[1] V. Segal and K. Raj, "An investigation of power transformer cooling with magnetic fluids," Ind. J. Eng. \& Mat. Sci., vol. 5, pp. 416-422, 1998.

[2] J. Patel, K. Parekh, and R. V. Upadhyay, "Prevention of hot spot temperature in a distribution transformer using magnetic fluid as a coolant," Int. J. Thermal Sci., vol. 103, pp. 35-40, 2016.

[3] R. Zanella, C. Nore, F. Bouillault, L. Cappanera, I. Tomas, X. mininger and J.-L. Guermond, "Study of Magnetoconvection Impact on a Coil Cooling by Ferrofluid with a Spectral/Finite-Element Method," IEEE Trans. Magn., vol. 54, no. 3, 4600104, March 2018

[4] R. Zanella, "Thermomagnetic Convection in Ferrofluids: Finite Element Approximation and Application to Transformer Cooling," PhD thesis, Paris-Saclay University, 2018. 